\title{
Dolutegravir drug-drug interactions
}

\author{
E Mondleki, MB ChB, HIV Dip Man; G Maartens, MB ChB, MMed \\ Division of Clinical Pharmacology, Department of Medicine, Faculty of Health Sciences, University of Cape Town, South Africa
}

Corresponding author: E Mondleki (enkosi.mondleki@uct.ac.za)

Dolutegravir is neither an inducer nor an inhibitor of metabolising enzymes, and it therefore has a low propensity to act as a perpetrator of drug-drug interactions. Clinically significant decreases in dolutegravir exposure occur when it is co-administered with strong inducers (e.g. rifampicin, efavirenz) of drug metabolising enzymes and drug efflux transporters for which dolutegravir is a substrate - this can be overcome by increasing the dose of dolutegravir from $50 \mathrm{mg}$ once daily to $50 \mathrm{mg}$ twice daily. Dolutegravir significantly increases metformin plasma exposure: one must not exceed metformin $500 \mathrm{mg}$ twice daily with concomitant dolutegravir. Divalent or trivalent cations chelate dolutegravir. Therefore, concomitant administration of dolutegravir with aluminium- and calcium-containing antacids should be avoided. Dolutegravir can be administered with calcium and iron supplements, provided these are taken together with food.

S Afr Med J 2021;112(3):194-195. https://doi.org/10.7196/SAMJ.2021.v112i3.16316

Clinically relevant drug-drug interactions (DDIs) with antiretroviral drugs are common: a prevalence of $20 \%-41 \%$ has been reported in high-income countries. ${ }^{[1-3]}$ There are few studies on antiretroviral DDIs from Africa, but the prevalence of clinically relevant DDIs ranged from $15 \%$ to $34 \%$ in three studies undertaken in Nigeria, Kenya and South Africa. ${ }^{[4-6]}$ When antiretroviral drugs are compounded by DDIs, this can result in increased toxicity when co-administered with strong inhibitors, or reduced efficacy when co-administered with strong inducers, which can increase the risk of the development of resistance. Some antiretroviral drugs can be the perpetrators of DDIs because they inhibit or induce drug-metabolising enzymes or drug transporters.

Dolutegravir is a second-generation integrase strand transfer inhibitor (INSTI), ${ }^{[7]}$ which is currently being rolled out as the preferred antiretroviral drug in first-line and second-line antiretroviral therapy (ART) in low- to middle-income countries.

Dolutegravir is rapidly absorbed, with a median time to maximum plasma concentration ranging from 0.5 to 2 hours. ${ }^{[8]}$ The terminal elimination half-life of dolutegravir is approximately 14 hours, which makes it suitable to be given once a day. The absorption of dolutegravir is increased when taken with meals, especially fatty meals: the dolutegravir area under concentration curve (AUC) increases from $33 \%, 41 \%$ and $66 \%$ when administered with low-, moderate- and high-fat meals, respectively, compared with a fasting state. ${ }^{[9]}$ However, these changes in dolutegravir exposure are not expected to affect safety or efficacy, and dolutegravir can be administered with or without meals. ${ }^{[9]}$

Dolutegravir is a substrate of the drug efflux pumps P-glycoprotein and breast cancer resistance protein (BCRP), and is primarily metabolised by UDP-glucuronosyltransferase $1 \mathrm{~A} 1$ (UGT1A1), together with a minor contribution from the cytochrome P450 enzyme CYP3A4. ${ }^{[10]}$ Dolutegravir is neither an inducer nor an inhibitor of metabolising enzymes, and it therefore has a low propensity to act as a perpetrator of DDIs. ${ }^{[1]}$ However, dolutegravir can be a victim of DDIs.

DDIs can be classified as either pharmacokinetic or pharmacodynamic based on the mechanism of the interaction. Pharmacokinetic interactions impact the absorption, distribution, metabolism or elimination of interacting drugs, whereas pharmacodynamic interactions result in synergistic, additive or antagonistic drug responses. ${ }^{[12]}$ In this review, we discuss pharmacokinetic DDIs of dolutegravir with other commonly used drugs. A summary of these DDIs with dolutegravir is given in Table 1.

\section{Dolutegravir pharmacokinetic DDIs}

The mechanism of action of INSTIs involves binding to magnesium at the active site of the integrase enzyme, preventing insertion of HIV viral DNA into host cell DNA. Therefore, the absorption of INSTIs can be reduced by chelation with divalent and trivalent metal cations. ${ }^{[13]}$ Administration of antacids containing magnesium and aluminium 2 hours after dolutegravir administration decreased dolutegravir AUC and minimum concentration ( $\mathrm{Cmin}$ ) by $26 \%$ and $30 \%$, respectively. Therefore, concomitant administration of dolutegravir and antacids should be avoided. Dolutegravir can be administered 2 hours before or 6 hours after antacids. ${ }^{[13]}$

People living with HIV, especially pregnant women, often take mineral supplements in combination with their antiretroviral medication. Plasma dolutegravir AUC and Cmin were reduced by $37 \%$ and $39 \%$, respectively, when co-administered with calcium carbonate under fasting conditions. ${ }^{[13]}$ Plasma dolutegravir AUC and $\mathrm{Cmin}$ were reduced by $54 \%$ and $57 \%$, respectively, when co-administered with ferrous fumarate under fasting conditions. ${ }^{[13]}$ Therefore, co-administration of dolutegravir and calcium or iron supplements under fasting conditions is not recommended. However, there is no clinically significant interaction when dolutegravir is co-administered with calcium or iron supplements when taken with meals. ${ }^{[13]}$

Clinically significant DDIs requiring a dose adjustment of dolutegravir have not been observed with inhibitors of UGT1A1, CYP3A4 or P-glycoprotein. ${ }^{[14]}$ However, clinically significant decreases in dolutegravir exposure occur when it is co-administered with strong inducers of the expression of genes encoding for drugmetabolising enzymes and drug efflux transporters, e.g. rifampicin, which induces P-glycoprotein, BCRP, UGT1A1 and CYP3A4. DDIs from strong inducers can be overcome by increasing the dose of dolutegravir from $50 \mathrm{mg}$ once daily to $50 \mathrm{mg}$ twice daily. ${ }^{[15,16]}$ Induction wanes over a few weeks, therefore the double dose of dolutegravir needs to be continued for 2 weeks after stopping the 


\begin{tabular}{|c|c|c|}
\hline Interacting drug & Effect of co-administration ${ }^{*}$ & Recommendation \\
\hline Metformin & $\uparrow$ metformin & Maximum metformin dose $500 \mathrm{mg}$ 12-hourly \\
\hline $\begin{array}{l}\text { Polyvalent cations (magnesium, iron, calcium, } \\
\text { aluminium, zinc), e.g. antacids, sucralfate, } \\
\text { supplements }\end{array}$ & $\downarrow$ dolutegravir & $\begin{array}{l}\text { Take dolutegravir either } 2 \text { hours before or } 6 \text { hours after } \\
\text { Calcium and iron can be co-administered with a meal }\end{array}$ \\
\hline $\begin{array}{l}\text { Anticonvulsants: carbamazepine, phenobarbital, } \\
\text { phenytoin }\end{array}$ & $\downarrow$ dolutegravir & $\begin{array}{l}\text { Avoid co-administration if possible (lamotrigine, } \\
\text { valproate, levetiracetam and topiramate can be used) or } \\
\text { double dolutegravir dose to } 50 \mathrm{mg} \text { 12-hourly }\end{array}$ \\
\hline Rifampicin & $\downarrow$ dolutegravir & $\begin{array}{l}\text { Double dolutegravir dose to } 50 \mathrm{mg} 12 \text {-hourly, or switch } \\
\text { rifampicin to rifabutin } 300 \mathrm{mg} \text { daily }\end{array}$ \\
\hline Efavirenz & $\downarrow$ dolutegravir & $\begin{array}{l}\text { Avoid co-administration if possible (rilpivirine can be } \\
\text { used) or double dolutegravir dose to } 50 \mathrm{mg} 12 \text {-hourly }\end{array}$ \\
\hline Nevirapine & $\downarrow$ dolutegravir & $\begin{array}{l}\text { Avoid co-administration if possible (rilpivirine can be } \\
\text { used) or double dolutegravir dose to } 50 \mathrm{mg} 12 \text {-hourly }\end{array}$ \\
\hline
\end{tabular}

inducing drug (e.g. after stopping rifampicin-based antituberculosis therapy).

Similarly, the inducing effects of efavirenz wane over a few weeks after switching from efavirenz to dolutegravir. Provided the viral load is suppressed, there is no need to adjust the dose of dolutegravir when switching from efavirenz to dolutegravir, because efavirenz concentrations remain therapeutic for the few days it takes for dolutegravir to reach therapeutic concentrations. However, if the viral load is not suppressed when switching from efavirenz to dolutegravir (e.g. when switching to dolutegravir in a second-line ART regimen owing to virological failure on an efavirenz-based first-line ART regimen), the dose of dolutegravir should be doubled for the first 2 weeks.

Dolutegravir is an inhibitor of organic cation transporter 2 $\left(\right.$ OCT2) ${ }^{[17]}$ resulting in elevations in serum creatinine levels through inhibition of active tubular secretion, but note that dolutegravir is not nephrotoxic. Metformin is a substrate of OCT2, therefore dolutegravir significantly increases metformin plasma exposure: metformin AUC and Cmax were increased by $79 \%$ and $66 \%$, respectively, when administered with dolutegravir $50 \mathrm{mg}$ daily. It is important not to exceed metformin $500 \mathrm{mg}$ twice daily with concomitant dolutegravir. ${ }^{[18]}$

\section{Summary}

- Dolutegravir is neither an inducer nor an inhibitor of metabolising enzymes. It therefore has a low propensity to act as a perpetrator of DDIs.

- Clinically significant decreases in dolutegravir exposure occur when it is co-administered with strong inducers (e.g. rifampicin, efavirenz) of drug-metabolising enzymes and drug efflux transporters for which dolutegravir is a substrate - this can be overcome by increasing the dose of DTG from $50 \mathrm{mg}$ once daily to $50 \mathrm{mg}$ twice daily.

- Dolutegravir significantly increases metformin plasma exposure: do not exceed metformin $500 \mathrm{mg}$ twice daily with concomitant dolutegravir.

- Divalent or trivalent cations chelate dolutegravir. Therefore, concomitant administration of dolutegravir with aluminium- and calcium-containing antacids should be avoided. Dolutegravir can be administered with calcium and iron supplements, provided these are taken together with food.
Declaration. None.

Acknowledgements. None.

Author contributions. EM wrote and revised the manuscript. GM assisted with the development and revision of the manuscript.

Funding. None.

Conflicts of interest. None.

1. Miller CD, El-Kholi R, Faragon JJ, Lodise TP. Prevalence and risk factors for clinically significant drug interactions with antiretroviral therapy. Pharmacotherapy 2007;27:1379-1386. https://doi.org/10.1592/ phco.27.10.1379

2. Marzolini C, Elzi L, Gibbons S, et al. Prevalence of comedications and effect of potential drug-drug interactions in the Swiss HIV Cohort Study. Antivir Ther 2010;15:413-423. https://doi.org/10.3851/imp1540 3. De Maat MMR, De Boer A, Koks CHW, et al. Evaluation of clinical pharmacist interventions on drug interactions in outpatient pharmaceutical HIV-care. J Clin Pharm Ther 2004;29:121-130. https://doi. $\operatorname{org} / 10.1111 / j .1365-2710.2003 .00541 . x$

4. Kigen G, Kimaiyo S, Nyandiko W, et al. Prevalence of potential drug-drug interactions involving antiretroviral drugs in a large Kenyan cohort. PLoS One 2011;6(2):e16800. https://doi.org/10.1371\%2Fjournal. pone. 0016800

5. Seden K, Merry C, Hewson R, et al. Prevalence and type of drug-drug interactions involving ART in patients attending a specialist HIV outpatient clinic in Kampala, Uganda. J Antimicrob Chemother 2015;70(3):33173322. https://doi.org/10.1093/jac/dkv259

6. Katende-Kyenda NL, Lubbe MS, Serfontein JHP, Truter I. Prevalence of drug-drug interactions of antiretroviral agents in the private health care sector in South Africa. S Afr Med J 2008;98(2):109-113.

7. Maartens G, Boffito M, Flexner CW. Compatibility of next-generation first-line antiretrovirals with rifampicin-based antituberculosis therapy in resource limited settings. Curr Opin HIV AIDS 2017;12(4):355358. https://doi.org/10.1097/coh.0000000000000376

8. Cottrell ML, Hadzic T, Kashuba ADM. Clinical pharmacokinetic, pharmacodynamic and drug-interaction profile of the integrase inhibitor dolutegravir. Clin Pharmacokinet 2013;52(11):981-994. https://doi. org/10.1007/s40262-013-0093-2

9. Song I, Borland J, Chen S, et al. Effect of food on the pharmacokinetics of the integrase inhibitor dolutegravir, Antimicrob Agents Chemother 2012;56(3):1627-1629. https://doi.org/10.1128\%2FAAC.05739-11

10. Zhang J, Hayes S, Sadler BM, et al. Population pharmacokinetics of dolutegravir in HIV-infected treatmentnaive patients. Br J Clin Pharmacol 2015;80(3):502-514. https://doi.org/10.1111/bcp.12639

11. Cattaneo D, Capetti A, Rizzardini G. Drug-drug interactions of a two-drug regimen of dolutegravir and lamivudine for HIV treatment. Expert Opin Drug Metab Toxicol 2019;15(3):245-252. https://doi.org/10.1 lamivudine for HIV treatme

12. Rathbun RC, Liedtke MD. Antiretroviral drug interactions: Overview of interactions involving new and Rathbun RC, Liedtke $\mathrm{MD}$. Antiretroviral drug interactions: Overview of interactions involving new and
investigational agents and the role of therapeutic drug monitoring for management. Pharmaceutics investigational agents and the role of therapeutic drug monitoring for management. Pharmaceutics
2011;3(4):745-781. https://doi.org/ $10.3390 \% 2$ Fpharmaceutics3040745

13. Song I, Borland J, Arya N, Wynne B, Piscitelli S. Pharmacokinetics of dolutegravir when administered with mineral supplements in healthy adult subjects. J Clin Pharmacol 2015;55(5):490-496.

14. Song I, Min SS, Borland J, et al. The effect of lopinavir/ritonavir and darunavir/ritonavir on the HIV integrase inhibitor S/GSK1349572 in healthy participants. J Clin Pharmacol 2011;51(2):237-242.

15. Song I, Borland J, Chen S, et al. Effects of enzyme inducers efavirenz and tipranavir/ritonavir on the pharmacokinetics of the HIV integrase inhibitor dolutegravir. Eur J Clin Pharmacol 2014;70(10):1173-1179.

16. Dooley KE, Sayre P, Borland J, et al. Safety, tolerability, and pharmacokinetics of the HIV integrase inhibitor dolutegravir given twice daily with rifampin or once daily with rifabutin. J AIDS J Acquir Immune Defic Syndr 2013;6(1)2:21-27. https://doi.org/10.1097/qai.0b013e318276cda9

17. Reese MJ, Savina PM, Generaux GT, et al. In vitro investigations into the roles of drug transporters and metabolizing enzymes in the disposition and drug interactions of dolutegravir, a HIV integrase inhibitor. Drug Metab Dispos 2013;41(2):353-361. https://doi.org/10.1124/dmd.112.048918

18. Song IH, Zong I. Borland J, et al. The effect of dolutegravir on the pharmacokinetics of metformin in healthy subjects. J Acquir Immune Defic Syndr 2016;72(4):400-407. https://doi.org/10.1097/qai.0000000000000983

Accepted 17 December 2021 